\title{
Effector: Energy Efficient Mobility Classification and State Based Route Creation
}

\author{
Sherine Santosh \\ Department of Computer Science and Engineering, AWH Engineering College, Calicut, India
}

\begin{abstract}
Mobility analysis of users with or without location determination using accelerometer sensor embedded within the user smartphone is energy efficient and provides real-time contextual information. The accelerometer measurements for mobility analysis of human beings presents its own challenges as users carry their smartphones differently and these measurements are dependent on body placement of the mobile phones. On demand remote data exchange for analysis and processing of measurements plays a key role in mobility state analysis using accelerometer sensor which is less energy efficient has higher network costs and is not real time. This method presents an energy efficient novel framework capable of identifying mobility state of the user and creating route maps based upon a probabilistic algorithm that neutralizes the effect of different smartphone on body placements and orientations to allow human movements to be more accurately and energy efficiently identified. In addition it tracks the location of the user and creates routes based on the mobility states. The use of embedded smartphone accelerometer and GPS without need for referencing historical data and accelerometer noise filtering is capable of identifying human mobility states in real time with a time constraint of 2 seconds based on which user paths can be generated. The method achieves an overall average high classification accuracy and saves energy to a great extent when compared to existing GPS only usage for route creations.
\end{abstract}

Keywords: Energy Efficient Mobility Classification and State Based Route Creation (EFFECTOR), Global Positioning System (GPS), Smartphone Accelerometer, Activity Log, Activity Chart, Route Maps

\section{Introduction}

Mobile Computing is a technology that allows transmission of data, voice and video via a computer or any other wireless enabled device without having to be connected to a fixed physical link. It is human computer interaction by which a computer is expected to be transported during normal usage. One of the applications of mobile computing is Mobility Based Services. Mobility awareness and mobility based services (MBS), in contrast to position or location based services (LBS) focus on mobility in the sense of how someone or something moves in the physical world to a pre-planned destination and covers ad hoc movement away from the current location. The emphasis is on the type of the mobility rather than on the location context, however these two may be combined in a complementary manner. . Mobility can be characterized at a low level as the rate of change of location in $(\mathrm{x}, \mathrm{y}, \mathrm{z})$ directions and velocity with respect to time. Mobility patterns of acceleration can be used to determine the travel or transportation mode of the user, i.e., the user is in a moving vehicle versus walking.

Mobility can be determined using smartphone sensors such as accelerometer and GPS. The first one is a non transceiver based sensor that directly measure physical world phenomena that relate to position and/or orientation changes. The accelerometer is the most valuable nontransceiver sensor used to provide the data for activity monitoring as it gives more information about movement forces. Accelerometer is used to measure the acceleration force a moving object. The second one is a transceiver based location signal sensor which require data exchange between multiple transmitters and receivers, determining the degree of physical exercise, the usage patterns for types of public and private transport, the time spent at a location all relate to human mobility profiling which constitutes one of the motivations for mobility based services. Adapting to the mobility information services dynamically based upon the travel mode in case where a pedestrian map is triggered after detecting walking shows safer places to cross roads whereas a motorist map focuses more on the main routes which is another motivational factor for MBS.

Accelerometer is an instrument that measures the acceleration force of an object be it a stationary object or a moving or a vibrating object. They can be embedded in any types of devices be it a smartphone, or vehicle or a laptop. Some of the uses of accelerometers are:

1. It can be used in car industry for detecting car crashes and deploying air bags instantaneously.

2. Widely used in smartphones to activate auto screen rotation on mobile devices.

3. Also used in gaming controls to use mobile screens for navigation without pressing the keys.

The Global Positioning System (GPS) is a space based navigation system that provides location and time information in all weather conditions, anywhere on or near the earth where there is an unobstructed line of sight to four or more GPS satellites. The system provides critical capabilities to military, civil and commercial users around the world. GPS satellites circle the earth twice a day in a very precise orbit and transmit signal information to earth. GPS receivers take this information and use trilateration to calculate the user's exact location. Essentially, the GPS receiver compares the time a signal was transmitted by a satellite with the time it was received. The time difference tells the GPS receiver how far away the satellite is. Now, with distance measurements from a few more satellites, the receiver can determine the user's position and display it on the unit's electronic map. 


\section{International Journal of Science and Research (IJSR) \\ ISSN (Online): 2319-7064 \\ Index Copernicus Value (2013): 6.14 | Impact Factor (2015): 6.391}

\section{Literature Survey}

There are several methods for detecting human activities. Electronic Pedometer [1] is a simple portable and electronic device used to assess physical activity. The device is used to determine the step count that a person takes by detecting the motion of the person's hands or hips. The step counts thus obtained can used to determine the distance travelled by user. This work could determine only step counts and no other mobility states could be determined.

User Annotated Acceleration Data [2] uses multiple wire free accelerometers are mounted on hoarder boards and worn simultaneously on different parts of the body to detect mobility states of user. Accelerometers sample acceleration values for various activities of the user during a specific time intervals. However since multiple accelerometers were used it caused practical difficulties for the user and could be used only in laboratory settings.

UbiFit Garden [3] uses on body sensing, activity inference and a novel personal, mobile display to encourage physical activity. It consists of three components fitness device, interactive application, glanceable display. This system consists of two separate entities MSP and mobile phones. Hence user has to maintain these two entities and hence maintenance overhead is slightly high and on body placements of MSPs are required for correct inference of activities.

Using every day GSM traces [4] and Parsimonious Mobility Classification using GSM and WiFi Traces [5] for mobility detection uses coarse grained GSM data from mobile phones to recognize high level properties of user mobility. These signals preserved the location privacy details of the user as Wifi and GSM signals do not use location details of the user for mobility detection. But these signals could classify only limited activities like dwelling, walking or driving and also resulted in decreased detection accuracy.

Mobility Based on GPS Data [6] infer peoples motion modes from their GPS logs. Mobility classification is performed by the segmentation, feature extraction, and inference processes. Though detection accuracy is high using GPS data, it reduces the battery life of to a great extent thereby increasing energy consumption.

Accelerometer Augmented Mobile Phone Localization (AAMPL) [7] is a framework that accepts the approximate physical location of a mobile phone, and augments it with context-aware logical localization. Hence it is a framework for combining physical and logical localization via real time classification of accelerometer readings. The drawback of this system was data exchange with remote servers reduced battery life and accelerometers were used only for detecting only limited states.

Wang [8] in his work identifies an Energy Efficient Mobile Sensing for Automatic User State Recognition (EEMSS) which is a sensor management scheme for mobile devices. The demerit of using this scheme are on body placement of accelerometer is required hence its limited use. Also it was used to identify only if object is moving and trigger other sensors for motion type identification.

Using mobile phones to determine transportation modes [9] focuses on transportation mode of an individual when outside, whether the user is stationary, walking, running, biking or in motorized transport. It targets applications for this fine grained transportation mode inference and location information. The limitations of this system are energy consumption is high as the system uses GPS along with accelerometer for classification of transportation modes. Moreover no backup measures were implemented for GPS like usage of alternate signals like wifi or GSM in case the GPS signals failed.

The above mentioned related papers had limitations like low detection accuracy and high phone battery power consumption. These drawbacks were overcome by the Energy Efficient Human Mobility Sensing (EHMS) [10] model. This model is used to classify the real time human mobility state using smartphones. The novel features of this research when compared to previous systems involving activity recognition was that it used extracted features from accelerometer data sensitive enough to classify activities with similar human patterns. This model permits similar activities such as motorized travel by bus and car to be classified at granular levels because the accelerometer data was more aligned to the activity.

\subsection{EHMS Architecture}

In this section we describe the system architecture of existing system. EHMS consists of two aspects. They are human mobility classification and optional user personalization.

Human Mobility Classification: Earlier systems used vertical y axis to detect mobility patterns. This was used as it presented the most differences because of the orientation of the device for identifying the human activities. In this system, all the three axes readings $\mathrm{x}, \mathrm{y}$ and $\mathrm{z}$ for a mobility state identification is used. This method of using all the axes classifies accelerometer data from a specialized subset of human mobility states including stationary with no movement and stationary with slight movements. In this classification process the activity details performed by the user would be already stored in his mobile phone whose values.

User Personalization: For similar activities performed by the user different mobility patterns are generated. In this process various variations of a user's activity need to be identified correctly. This involves personalizing the mobility sensing model for a specific activity. The user performs an activity continuously for 14 seconds so that range values can be determined for the activity being performed by the user. 


\section{International Journal of Science and Research (IJSR) ISSN (Online): 2319-7064 \\ Index Copernicus Value (2013): 6.14 | Impact Factor (2015): 6.391}

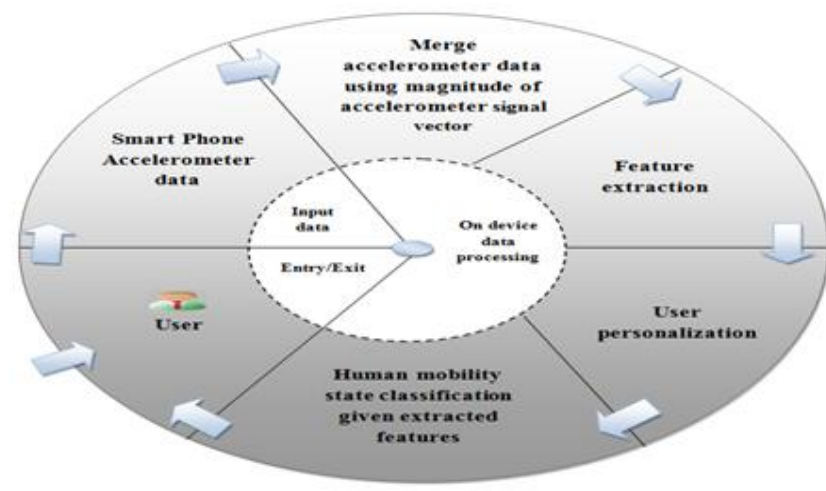

Figure 1: Architecture of energy efficient human mobility sensing for mobility detection.

\section{Problem Definition}

The EHMS model used a standalone accelerometer framework for detecting human activities. One major drawback of this system was that it could not be used for determining the location or position of the user. This standalone framework could be further extended or enhanced to support various mobility based service applications in a better energy efficient manner. As the sensor used was a single accelerometer this model was incapable of identifying the user position or location. These limitations were looked upon and a new method was introduced to identify the position of the user along with their mobility states.

\section{New Methodology}

To overcome the above mentioned drawbacks of the existing method which was EHMS a new system has been introduced called Effector which is Energy Efficient Mobility Classification and State Based Route Creation. The salient features of the new system that overcomes the limitations of the existing system are that this new system is an energy efficient mobility classification model that integrates the energy efficient human mobility sensing model along with the GPS sensors. This model can be used to identify the mobility states of users using an embedded smartphone accelerometer. It can also be used to identify the user locations with the help of GPS sensors inside the user phone.

\subsection{EFFECTOR System Architecture}

Effector consists of six following phases. They are;

- Activity Detection

- Feature Extraction

- Activity Personalization

- Mobility Classification

- GPS based Location Tracking

- State based Route Creation

The sensors like accelerometer and GPS are present inside the user's mobile phone. User activates the accelerometer and performs an activity. In the activity detection phase the accelerometer starts capturing the activity readings along the $\mathrm{x}, \mathrm{y}$ and $\mathrm{z}$ directions. Based on these accelerometer readings a parameter called magnitude of accelerometer (MASV) is determined. In feature extraction phase several parameters are extracted based on the determined MASV values. If the activity performed by the user is a new activity whose details are not stored in user's phone then activity personalization is performed. This is done to determine the threshold ranges for the new activity being performed. If the user performs an already existing activity whose details are known, then mobility classification is done. Once the mobility state of the user is identified user gets his activity details from the activity log and activity chart. Based on the identified mobility state if the detected activity is walking, running or jogging then user activates the GPS sensor inside his mobile phone. The GPS sensor tracks the user's location and position in a switch on and switch off mode continuously for fixed time intervals. The location details thus collected can be used to create route maps for users like pedestrian paths or jogging paths based on the identified mobility state.

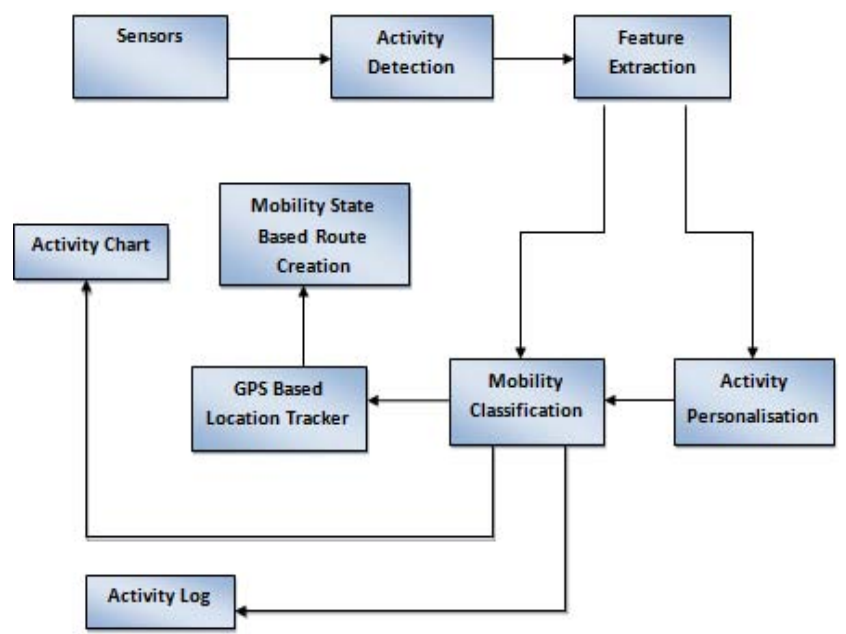

Figure 2: System Architecture of Effector

\subsection{Activity Detection}

Mobility can be determined using smartphone sensors like GSM, Wi-Fi, GPS, accelerometer etc. Of the above, the sensor that consumes the least amount of battery power of mobile phone is the accelerometer. Hence the proposed system uses accelerometers for detecting activities of the user. These sensors directly measure the physical world phenomena that relate to position and/or orientation of objects. Accelerometer is an instrument that measures the acceleration forces of an object be it a stationary object or a moving or vibrating object. The accelerometer has many advantages over transceiver based location signal sensors such as they have low energy consumption when compared to GPS sensors. Also sensor readings are continuously available with the accelerometer as compared to transceiver based sensors which are susceptible to signal obstructions or noise. Hence in this activity detection phase the user activates the accelerometer sensor in his smartphone and performs an activity by holding the smartphone or placing it on any part of his body. The accelerometer senses the activity performed by the user and starts capturing the activity readings in the $\mathrm{x}, \mathrm{y}, \mathrm{z}, \mathrm{z}$ directions. 


\subsection{Activity Feature Extraction}

In this phase of the proposed research, the focus is on using these $3 \mathrm{D}$ readings captured by the accelerometer for a user activity and determines a parameter called magnitude of the accelerometer signal vector (MASV). This MASV is used to combine $(\mathrm{x}, \mathrm{y}$, and $\mathrm{z})$ readings regardless of the smartphone orientation. Fast growth of smartphone market over the last few years has made the mobile phone an appealing platform for context sensitive and location based applications. However, common issue for all these applications is high power consumption and subsequent lack of battery life, not allowing such application to run for extended periods of time. In this proposed research the focus is on classification of human mobility states, concretely stationary and active. This information is further used for location monitoring with additional power saving methods for creation of transportation or pedestrian paths. The smartphone embedded accelerometer patterns are detected based on the vertical y axis. This axis presented the most differences because of the orientation of the device during the experiments. However for similar activities with different smartphone on body placements this vertical axis alone is not sufficient for determining the activity states and so the above parameter is determined.

Using the magnitude of the accelerometer signal vector six other computation features are extracted as classifiers from the accelerometer readings for a given human mobility state. These extracted features are light to calculate and can be extracted using a comparatively low sampling rate such as $4 \mathrm{~Hz}$. The features are selected based on the MASV wave patterns generated for different activities. The troughs and peaks better characterizes the wave patterns than the standard local/global maxima/minima. Different human activity states such as stationary vs. walking are classified using the peak and trough features, but similar activities such as jogging vs. walking produced incorrect results using only these peak and trough values. Hence for identifying similar activities features like mm, Pmm and Tmm are extracted. These features were extracted by setting the extraction window as 2 seconds.

The different parameters that are determined as part of the activity detection and feature extraction phases are as follows:

- MASV : calculated as square root of $(x 2+y 2+z 2)$ accelerometer readings

- (P) : No of peaks.

- (T) : No of troughs

- $\mathrm{T}_{\mathrm{PT}}$ : Total no. of peaks and troughs

- mm : difference between the maximum peak and maximum trough values and their corresponding minimum values

- $\mathrm{P}_{\mathrm{mm}}$ : difference between the maximum and minimum peak values

- $\mathrm{T}_{\mathrm{mm}}$ : difference between maximum and minimum trough values.

\section{4}

\section{Activity Personalization}

This personalization phase is the next step once the above features have been extracted for an activity. For similar activities different human mobility patterns are generated by different users. The personalization algorithms must be able to adapt to the various variations while a user is performing an activity. It should be able to identify walking activity for one group and must be able to identify jogging activity for another group. Personalization is the practice of dynamically tailoring an application or site to the wants and needs of each user. The initial step for this personalization involves reconfiguring the algorithm based on the smartphone accelerometer data gathered for the specific activity. This personalization process is a one time process where the user performs the activity continuously for 14 seconds. Activity personalization phase involves deriving the following: $\mathrm{TP} \mathrm{T}$ range estimation, Pmm range, and Tmm range. Hence in this phase the user customizes the personalization algorithms for each type of mobility states and stores the values in the phone for further activity classification process. Personalization algorithm is used for determining the threshold ranges for a new activity performed by the user.

The design of the algorithm is as follows:

1. Initialize feature extraction period as $2 \mathrm{sec}$.

2. Initialize sampling frequency as $4 \mathrm{~Hz}$.

3. Perform an activity continuously for $14 \mathrm{sec}$.

4. Extract peaks and troughs at interval of $2 \mathrm{sec}$.

5. Aggregate TPT values for all seven iterations

6. Check if sum for 2 or 3 consecutive TPT values is greater than or equal to 75 percent.

7. If true, TPT range for an activity is between the corresponding min and max TPT values.

\subsection{Mobility Classification}

In the mobility state classification phase with the help of the extracted features from the accelerometer sensor, the next step is to identify the human mobility state of a user. This is determined with the help of the threshold values that are personalized for each user which are $\mathrm{T}_{\mathrm{PT}}$ ranges, $\mathrm{mm}$ ranges, $\mathrm{P}_{\mathrm{mm}}$ ranges and $\mathrm{T}_{\mathrm{mm}}$ ranges. Accelerometers embedded inside the smartphone are used to identify the mobility state of an user once the determined values like $\mathrm{mm}, \mathrm{T}_{\mathrm{PT}}, \mathrm{P}_{\mathrm{mm}}$ and $\mathrm{T}_{\mathrm{mm}}$ which are calculated every two seconds fall within the above estimated range values. The different human mobility states include stationary with no movement e.g., smartphone resting on a table, stationary with slight movements (sitting, lying down, and standing) and in-motion (walking, jogging, cycling, motorized movement including travel by bus, light rail train, underground train, taxi, and car). Once the mobility state has been identified based on the activity state the activity chart is displayed to the user stating about his mobility. If the user wishes, based on the mobility state routes can be created for the user using GPS based location tracking system. The classification algorithm is used for identifying the mobility state of the user. The design of algorithm is as shown: 


\section{International Journal of Science and Research (IJSR) \\ ISSN (Online): 2319-7064 \\ Index Copernicus Value (2013): 6.14 | Impact Factor (2015): 6.391}

1. Initialize feature extraction period as $2 \mathrm{sec}$.

2. Initialize sampling frequency as $4 \mathrm{~Hz}$.

3. Perform an activity.

4. Extract the peaks and troughs at interval of 2 sec.

5. Determine the values for $\mathrm{mm}, \mathrm{T}_{\mathrm{PT}}, \mathrm{P}_{\mathrm{mm}}$ and $\mathrm{T}_{\mathrm{mm}}$ for the activity performed.

6. Verify if the value for $\mathrm{mm}, \mathrm{T}_{\mathrm{PT}}, \mathrm{P}_{\mathrm{mm}}$ and $\mathrm{T}_{\mathrm{mm}}$ falls within the max and min threshold values.

7. If true, current activity is the mobility state stored in user phone.

\subsection{GPS Based Location Tracking}

The mobility state classification phase determined with the help of the features extracted from the accelerometer helps to identify the mobility state of the user. These results can be further used by the user to create routes based on the activity states with the help of GPS location based sensors embedded within the user's mobile phone. This integration of the EHMS model with the GPS for determining pedestrian paths, jogging paths and transportation paths saves energy to a great extent when compared to route creations currently with the help of GPS only. This integration also helps to determine the location of users in addition to the mobility states. Hence the limitations of the EHMS standalone model are addressed using this integration scheme. As part of the integration system, the mobility states of the users are determined initially using the energy efficient human mobility sensing model. The mobility states as to whether the users is walking, running, stationary etc. is determined by using accelerometer sensor readings and features extracted from the sensor for mobility classification.

Once the mobility state of the user is identified as walking, running or jogging, the initial and destination points of the travel is established. This is followed by automatically switching on the GPS in the users mobile phone. Once the GPS is switched on the location of the user is identified at that particular point. The location details of that particular user position are stored and the GPS is switched off . The user keeps on moving and after a particular interval of time the GPS is switched on again and the user location at that point is determined and stored. The GPS is switched off again. The same process is repeated for a sufficient interval of time. Thus as the user moves the location of the user is tracked at different points in an on and off manner using the GPS. The locations thus determined are collected and a path is generated connecting the start and end points along the intermediate locations determined. The pedestrian path thus determined is plotted on a Google map.

\subsection{State Based Route Creation}

Once the mobility states of users have been identified route maps can be created for users. The route maps may be pedestrian paths or jogging paths depending on the identified mobility state. These route maps are created using embedded GPS sensors inside the user phone. The advantage of such route maps are they can be used by the users for their customized uses or can be monitored by an admin persons like trainers or government bodies planning for urban development. The route creation algorithm is used to create route maps for users based on their identified mobility states. This is done with the help of GPS sensor inside the user phone. The below steps describe the design of the algorithm.

1. Determine the mobility state of user

2. Check if the mobility state is walking, jogging or running

3. If true, turn on GPS in the user phone.

4. Identify and store location details of the user at that point.

5. Turn off the GPS in the user phone.

6 . Repeat step 3 through step 5 at time interval of 2 minute until user reaches his destination.

7. Collect the stored location details from the start point to the destination point.

8. Create route map for a pedestrian path with the connected locations.

9. For states other than walking, running and jogging do not turn the GPS on.

\section{Result}

This integration model specified in the proposed system determines both the mobility state and location of the user. It is an energy efficient scheme which consumes lesser battery power of phone when compared to standalone GPS sensor used for creating user routes. In the above figure the $\mathrm{X}$ axis denotes the different user paths created and the $\mathrm{Y}$ axis denotes the energy consumption of the phone. The values mentioned are rough estimates to demonstrate energy saving of the new scheme. As the new scheme creates user routes using GPS sensor in a switch on and switch off mode user phones consume lesser battery power. The proposed system also motivates user to perform physical activities as the user is able to get his activity details in logs and charts. The activity detection accuracy is comparatively higher as accelerometer is used for activity detection before user routes are created.

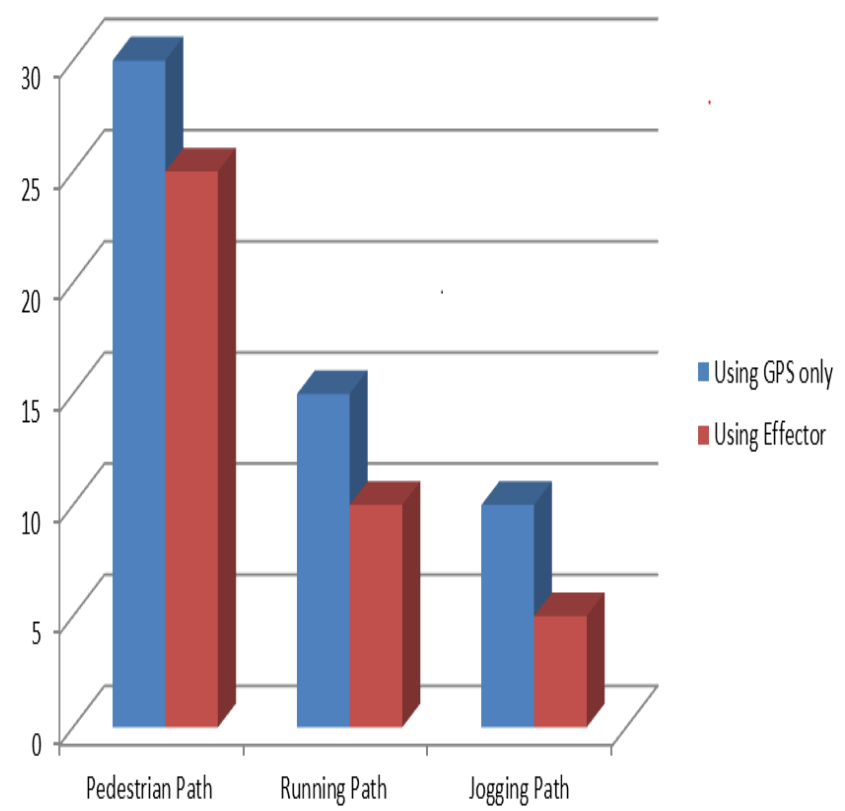

Figure 3: Comparison of energy consumption of GPS only system and Effector system 


\section{International Journal of Science and Research (IJSR) \\ ISSN (Online): 2319-7064 \\ Index Copernicus Value (2013): 6.14 | Impact Factor (2015): 6.391}

\section{Conclusion}

Effector presents novel frameworks that can energy efficiently identify human movements and location in real time. It neutralizes the effect of different smartphone on body placements and orientations for mobility classification as this model is able to identify the human mobility states irrespective of phone positions. It also creates routes like pedestrian paths or jogging paths for users based on mobility states saving battery consumption of the user phone to a great extent. Hence the proposed system can in addition use the inferred mobility states and create routes like pedestrian paths or jogging paths using GPS based location tracking system in an energy efficient manner. Position determination alone cannot differentiate between some human transport modalities or activities. Additional types of mobility context, other than location need to be sensed to classify these states. Hence our scheme combines acceleration values with location determination. Thus the new proposed system provides a real time human mobility state classification algorithm without need for referencing historical data. It also provides classification of the human mobility state regardless of the smartphone alignment and on body placement. Light weight accelerometer data feature extraction is another added advantage of this proposed scheme. Also there is no need for a remote server connection for computational purposes as all processing is performed within the smartphone. The proposed model is also relatively insensitive to noisy data. This scheme classifies human mobility states using probabilistic algorithms and feature extraction process on the smartphone accelerometer data. Location details are determined along with activity details which is another advantage of this proposed scheme. Thus the new classification and route creation scheme is energy efficient as it uses a switch on switch o mechanisms for route creation.

\section{Acknowledgements}

I am thankful to our beloved principal of AWH College of Engineering, Prof. Shahir V K and our respected Head of the Department of Computer Science and Engineering $\mathrm{Mr}$. Gireesh $\mathrm{T} \mathrm{K}$, for their parental guidance and support. I would like to thank our project co-coordinator Mrs. Divya M, Asst. Professor, Department of Computer Science \& Engineering, for giving me innovative suggestions and assisting in times of need. I gratefully acknowledge the excellent and incessant help given by my project guide Ms. Anjana T. K, Asst. Professor, Department of Computer Science \& Engineering, to incite this work. I am thankful for her valuable guidance and enduring encouragement provided throughout this study.

\section{References}

[1] Crouter, S., Schneider, P., Karabulut, M., and Bassett JR, D. 2003. Validity of 10 electronic pedometers for measuring steps, distance, and energy cost. Med. Sci. Sports Exercise 35, 8, 1455.
[2] L. Bao and S. S. Intille. Activity recognition from user-annotated acceleration data. In Proceedings of the 2nd International Conference on Pervasive Computing (PERVASIVE), volume 3001 of Lecture Notes in Computer Science, pages 1-17. Springer-Verlag, 2004.

[3] S. Consolvo, D. W. McDonald, T. Toscos, M. Y. Chen, J. Froehlich, B. Harrison, P. Klasnja, A. LaMarca, L. LeGrand, R. Libby, I. Smith, and J. A. Landay. Activity sensing in the wild: a field trial of UbiFit garden. In CHI '08: Proceeding of the twentysixth annual SIGCHI conference on Human factors in computing systems, pages 17971806, New York, NY, USA, 2008. ACM.

[4] T. Sohn, A. Varshavsky, A. LaMarca, M. Y. Chen, T. Choudhury, I. Smith, Consolvo, J. Hightower, W. G. Griswold, and E. de Lara. Mobility detection using everyday GSM traces. In Proceedings of the 8th International Conference on Ubiquitous Computing (Ubicomp), pages 212-224, 2006.

[5] M. Mun, D. Estrin, J. Burke, and M. Hansen. Parsimonious mobility classication using gsm and wifi traces. In Proceedings of the 5th International Conference on Embedded Networked Sensor Systems (SenSys), pages 1-5, 2008.

[6] Y. Zheng, Q. Li, Y. Chen, X. Xie, and W.-Y. Ma. Understanding mobility based on gps data. In Proceedings of the 10th international conference on Ubiquitous computing, pages 312-321, 2008.

[7] A. Ofstad, E. Nicholas, R. Szcodronski, and R. R. Choudhury, AAMPL: Accelerometer augmented mobile phone localization, in Proc. 1st Int. Conf. Mobile Entity Localization Tracking GPS-Less Envir., 2008, pp. 1318.

[8] Y. Wang, J. Lin, M. Annavaram, Q. A. Jacobson, J. Hong, B. Krishnamachari, and N. Sadeh, A framework of energy efficient mobile sensing for automatic user state recognition, in Proc. 7th Int. Conf. Mobile Syst., Appl., Serv., 2009, pp. 179192

[9] S. Reddy, M. Mun, J. Burke, D. Estrin, M. Hansen, and M. Srivastava, Using mobile phones to determine transportation modes, ACM Trans. Sensor Netw., vol. 6, no. 2, pp. 127, 2010.

[10] Thomas Olutoyin Oshin, Stefan Poslad, Zelun Zhang, "Energy Efficient Real-Time Human Mobility State Classification Using SmartPhones, " In IEEE Transactions on Computers., vol. 68, no. 6, pp. 16801693, 2015

\section{Author Profile}

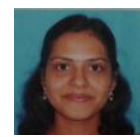

Sherine Santosh received the B.E degree in Computer Science and Engineering from C.S.I College of Engineering, Tamil Nadu in 2005. Later was working as a Quality Analyst in an industrial firm related to payments and mobile banking. Currently is pursuing final year Master's Degree in Computer Science and Engineering from AWH College of Engineering, Kozhikode. Key areas of interest include mobile technology and wireless networks using mobile platforms. Presently working on understanding the related researches and implementing the effector project as part of my thesis work. 